Reprod. Nutr. Dévelop., 1982, 22 (6), 889-904.

\title{
Déterminisme génétique de l'inversion du sexe femelle accompagnant le croisement de deux sous-espèces d'ldotea balthica (Pallas). II. - Résultats concernant le phénotype bilineata-lineata et son association avec le phénotype alba- fusca
}

\author{
Emmanuelle LEGRAND-HAMELIN, J.-J. LEGRAND
}

avec la collaboration technique de C. LE BOTE et G. HEILY

Laboratoire de Physiologie et Génétique des Crustacés, E.R.A. CNRS $n^{\circ} 230$, 40, avenue du Recteur Pineau, 86022 Poitiers Cedex

Summary. Genetic determinism of the female sex inversion accompanying the crossing of two subspecies of Idotea balthica (Pallas). II. - Results on the bilineata-lineata phenotype and its association with the albafusca phenotype.

The $O$ in $l . b$. is heterogametic (WZ) and the $\sigma^{*}$ homogametic (ZZ). The loci of the genes which produce the colored albafusca (A) and bilineata-lineata (BL) phenotypes are carried by the $W$ sex chromosome. Sex inversions are rare in I. b. basteri (ba), but more frequent in $\%$. $b$. tricuspidata (tr.). When these two subspecies are crossed, the rate of neo- $\sigma^{*} \sigma^{*}\left(=\mathrm{t}_{\mathrm{i}}\right)$ is considerably increased. The $/ . b$. tr. A or uniformis (U) $(=$ wild type) used in this study either came from the offspring $\left(t_{i}=27 \mathrm{p} .100\right.$ in five generations) of one gravid $q$ collected in La Rochelle (LR) or were collected on Oleron island (OI) and in Pirou (P) (Manche). The 1. b. ba BL came from either Marseille (M) or Racou (R) (Pyrénées-Orientales).

We used the $\chi^{2}$ test or the Mann-Whitney test at level 0.05 to compare the results of different crosses.

1) The crosses $0^{*} \operatorname{tr} U \times \&$ ba $\mathrm{BL}_{1}(\mathrm{M})$ gave a high $\mathrm{t}_{\mathrm{i}}(72$ to $100 \mathrm{p} .100)$ with all the (LR) males. When 90 p. 100 of the $\operatorname{tr}(\mathrm{OI})$ males and $94 \mathrm{p} .100$ of the $\operatorname{tr}(\mathrm{P})$ males were crossed with $b a \mathrm{BL}$ females, the $\mathrm{t}_{\mathrm{i}}$ rose from 26 to $100 \mathrm{p}$. 100 . Many of the ba BL females of (M) and $(R)$ lineages (excluding $B L_{1}$ and $B L_{4}$ from the $20 \%$ o tested) crossed with a tr $U$ (LR) ơ gave no neo-male.

2) The crosses neo- $\sigma A(L R) \times \odot B L_{1}(M)$ also gave a high $t_{i}(93 p$. 100) in the $B L$ phenotype $(q: W Z)$, and a low $t_{i}$ in the $A B L$ phenotype (corresponding to $W W q Q$ ) and the A phenotype ( $q: W Z$ ).

3) In the backcrosses between a same $A B L$ from (2) and two $U$ males, $\operatorname{tr}$ then $b a$, the penetrance of the $A$ gene was reduced exclusively in neo-males. In some successive crosses with the $b a O^{*}$, the $A t_{i}$ was higher than with the $\operatorname{tr}$ male but the $B L t_{i}$ were equivalent. In other crosses, the $B L t_{i}$ decreased but the $A t_{i}$ did not change significantly.

4) In the crosses neo- $O W_{B L} Z \times \propto W_{B L} Z$, the $t_{i}$ of $W W \circ \circ$ was significantly lower than predicted : $1 / 3$. This result emphasizes the lower inversion of WW females.

5) In $F_{1}$, the $t_{i}$ of $\sigma^{\prime} \operatorname{tr} U(L R) \times \&$ ba $B L(R)$ was 0 ; in $F_{2}$, the $t_{i}$ was zero or very low. In $\mathrm{F}_{1}$, the $\mathrm{t}_{\mathrm{i}}$ of $\sigma^{\prime} \operatorname{tr} \mathrm{U}(\mathrm{P}) \times \&$ ba $\mathrm{BL}_{4}(\mathrm{M})$ was very low $\left(2.6 \mathrm{p}\right.$. 100); the $\mathrm{t}_{\mathrm{i}}$ in $\mathrm{F}_{2}$ varied 
from 0 to 45 p. 100 and were significantly different than those of the preceding $F_{2}$ type.

These results led to the following conclusions.

- The (1), (2) and (3) crosses show that the sex inversion of ba/tr genetic hybrid females was controlled by hereditary characters which were present on the one hand in all populations of the $\operatorname{tr}$ subspecies and, on the other, in the $\mathrm{BL}_{1}$ and $\mathrm{BL}_{4}(\mathrm{M})$ lineages, but which seemed independent of the $B L$ mutation since many ba $B L$ females crossed with a tr male gave no neo-male.

- The (4) crosses show the importance of the $W$ chromosome in sex determination ; its heterologous segment carried a major female determinant, a double dose of which decreased the possibility of sex inversion.

- The (3) crosses show that the reduction of $A$ gene penetrance was always associated with sex inversion in the genetic hybrid females. This gene may be a part of the female determinant. The reduction of $A$ penetrance was controlled by some partly sex-linked and/or autosomal genes different from those which controlled the sex inversion of $B L$ hybrid females. We believe that sex inversion is conditioned by :

a) the valence of the $W$ sex chromosome which varies with the subspecies, the population and the mutations carried by the chromosome ;

b) some partly sex-linked and/or autosomal genes acting as a modifier of the $W$ valence.

- The results on the $F_{2}$ (5) suggest: (1) that many ba BL females had a strong $W$ valence and dominant modifier genes which almost entirely inhibited sex inversion (in inbreeding and outbreeding) and (2) that $B L_{4}$ (and $B L_{1}$ ) females had a weak $W$ valence but dominant modifier genes which inhibited inversion in inbreeding and in $F_{1}$ crosses with a $P$ male; the variable and rather high $t_{i}$ of the $F_{2}$ depend on favourable recombinations of the $b a / t r$ modifier genes.

- From these genetic results and experiments, sex determination in /sopoda Crustacea appears to be polyfactorial ; it seems to be based on a repression of the sex genes carried in each sex by the $Z$ chromosome and autosomes which induce differentiation of the other sex. In a male, the repressor is the androgenic hormone; in a female it may be a primary product of the heterologous segment of the $W$ chromosome.

\section{Introduction.}

L'étude de la transmission de caractères de coloration du corps a permis à l'un de nous (Tinturier-Hamelin, 1963) de montrer que la femelle d'/dotea balthica est hétérogamétique (WZ), le mâle étant $Z Z$. Ce résultat a pu être confirmé par l'emploi de néo-mâles expérimentaux (Legrand-Hamelin, 1977).

Dans un travail précédent (1982, sous-presse), nous avons montré que le croisement de deux des sous-espèces marines d'/dotea balthica : /. b. tricuspidata (Atlantique et Manche) et $/$. $b$. basteri (Méditerranée) pouvait, dans certains cas, renforcer considérablement l'inversion des femelles en néo-mâles, processus déjà connu comme intervenant plus fréquemment dans les populations naturelles (et d'élevage) d' $\%$. b. tricuspidata que dans celles d' $\%$ b. basteri (TinturierHamelin, 1963).

Nous avons utilisé, dans notre précédent travail de 1982, des lignées où I'hétérochromosome $W$ de la femelle ou du néo-mâle est marqué par des gènes non allèles conditionnant la coloration du corps (albafusca $=A$, porté par le segment impair, et bilineata $=B$, situé sur le segment pair mais, en l'occurence, strictement lié à $W$ ), ainsi que des individus dont la coloration répond au type 
sauvage uniformis $(=U)$. Le taux $d^{\prime}$ inversion sexuelle $\left(t_{i}\right)$ des femelles $s^{\prime}$ est montré variable en fonction des lignées et du sens du croisement.

Dans le croisement: $q$ basteri $A \times \sigma$ tricuspidata $U$, le $t_{j}$, généralement très bas en $F_{1}$, s'accroît par des croisements en retour successifs renforçant le génotype tricuspidata, jusqu'à atteindre le niveau caractérisant la lignée tricuspidata d'origine. Le renforcement du génotype basteri conduit au résultat inverse. Dans les croisements entre des femelles tricuspidata $A$ et des mâles basteri $U$ appartenant à certaines lignées, I'expressivité du phénotype $A$ est réduite : ce processus n'intervient que chez les néo-mâles et provoque généralement une augmentation de leur taux. Le croisement: $U$ montre que seules certaines femelles sont favorables à l'inversion sexuelle de leurs filles; les taux d'inversion sont alors plus élevés que ceux observés chez les hybrides $A$ où l'expressivité de ce phénotype est complète.

Dans certains croisements sont apparus des néo-femelles $U$ (homogamétiques : ZZ). Ces résultats nous ont conduits à considérer le déterminisme de l'inversion du sexe femelle comme polyfactoriel, mettant en jeu, d'une part, un ou plusieurs gène(s) majeur(s) déterminant le sexe femelle, porté(s) par l'hétérochromosome $W$ et étroitement lié(s) au locus albafusca, d'autre part, des modificateurs principalement autosomiques, peut-être également partiellement liés au sexe, agissant en inhibiteurs du (ou des) déterminant(s) femelle(s). Dans le présent travail, ont été utilisées des lignées où le chromosome $W$ de la femelle, ou du néo-mâle, est marqué par l'association des mutations bilineata et lineata (BL). Le locus de $L$, comme celui de $B$, est situé sur le segment pair de $W$. Dans certaines lignées, $B$ et $L$ peuvent être disjoints par crossing-over et se comportent comme partiellement liés au sexe. Dans les lignées utilisées ici, l'association $B L$ ne peut être rompue par suite de la présence d'un suppresseur de crossing-over et cette situation empêche tout échange entre parties homologues des hétérochromosomes (Tinturier-Hamelin, 1963). L'emploi de telles lignées va nous permettre d'appuyer et de préciser certaines de nos hypothèses précédentes.

\section{Matériel et méthodes.}

Rappelons que les femelles d'idotées ne possèdent pas de réceptacle séminal et doivent, de ce fait, être fécondées avant chaque ponte.

Les I. b. basteri de phénotype bilineata-lineata (BL) utilisées proviennent, d'une part, de $10 \%$ BL récoltées à Marseille, au lieu dit La Pointe Rouge (elles sont issues de la même population que celles utilisées dans le précédent travail), d'autre part, de 3 우 BL récoltées au Racou, près d'Argelès-sur-Mer (PyrénéesOrientales). Ces femelles n'ont donné, en inbreeding, qu'un taux très faible de néo-mâles : 1 néo- $O^{*}$ pour $330 \%$ $Q \mathrm{BL}=0,29 \mathrm{p} .100$ en 5 générations en ce qui concerne les femelles marseillaises, aucun néo- $\sigma^{*}$ contre $48 \%$ O BL pour les femelles du Racou croisées avec un mâle uniformis de même origine.

Les mâles basteri uniformis proviennent de la même population marseillaise et, plus précisément, de la $\$ A_{9}$ qui, en 5 générations, n'a donné aucun néomâle. 
Les I. b. tricuspidata proviennent, soit d'une lignée (déjà utilisée dans le précédent travail) issue d'une $q$ albafusca $A_{1}$ trouvée gravide à La Rochelle (Charente-Maritime) et ayant fourni, au fil de 5 générations, un taux constant de néo-mâles (27 p. 100), soit d'individus prélevés dans les populations naturelles de l'île d'Oléron (Charente-Maritime) et de Pirou-Plage (Manche).

Dans l'exposé des résultats, chaque descendance sera représentée par un rapport $(R)=$ néo- $\sigma^{*} O^{\prime} / Q$ Q de même phénotype coloré, $d^{\prime}$ où l'on peut déduire le taux d'inversion des femelles génétiques: $t_{i}=$ (néo- $\sigma^{*} \sigma^{*} /$ néo- $\sigma^{*} \sigma^{\prime}+\phi$ ) $\times 100$. Les analyses statistiques ont été effectuées à partir des rapports $R$ à l'aide du $\chi^{2}$ ou du test non paramétrique de Mann-Whitney (in Faverge, 1962), au niveau 0,05 .

\section{Résultats.}

1) $F_{1} \propto B L_{1}$ basteri (Marseille) $\times$ o $U$ tricuspidata (rochellais).

Cinq croisements de ce type (utilisant des femelles et des mâles différents) ont donné, pour le phénotype $B L$, des rapports $R$ : 10/0 46/5 13/1 29/11 39/1. Cet ensemble est hétérogène $\left(\chi^{2}=13,98-\mathrm{d} . \mathrm{I}=4\right)$. Le taux de néo- $\sigma^{*} \mathrm{O}^{*}$, très élevé, compris entre 72,5 et 100 p. 100 , est significativement différent de celui des néo- $\sigma^{\prime}$ ơ A dans la lignée tricuspidata rochellaise.

2) Corrélation entre inversion du sexe et phénotype BL.

Afin de voir si le taux élevé d'inversion constaté dans tous les croisements précédents utilisant la lignée $B L_{1}$ de Marseille était généralisable, nous avons croisé avec des mâles $U$ de La Rochelle les 9 autres femelles $B L$ récoltées à Marseille, ainsi que 10 femelles $B L$ issues des femelles récoltées au Racou. Ces dernières n'ont donné aucun néo- $\sigma^{\prime \prime}(0 / 226 \%$ BL). II en a été de même de $6 \%$ \% marseillaises $(0 / 222 \% \bigcirc \mathrm{BL})$, tandis que 2 autres ont donné chacune 1 néo- $O^{*}$ $B L(R=1 / 28$ et $1 / 24)$; enfin, la dernière $Q\left(B L_{4}\right)$ a donné un rapport $R=$ $30 / 28$, soit 53 p. 100 de néo- $O^{*} O^{*} \mathrm{BL}$.

3) $\mathrm{F}_{1} \bigcirc \mathrm{BL}_{1}$ basteri (Marseille) $\times$ néo- $\mathrm{O}^{\prime} \mathrm{A}$ tricuspidata (rochellais).

Quatre croisements de ce type (utilisant des femelles et des mâles différents) ont été réalisés. L'apparition de 3 phénotypes colorés: albafusca, bilineatalineata et albafusca-bilineata-lineata $(\mathrm{ABL})$ est prévue par l'équation (dont la sexratio théorique est de $1 \sigma / 3 \%$, les mâles $(Z Z)$ étant uniformis $(U)\}$ :

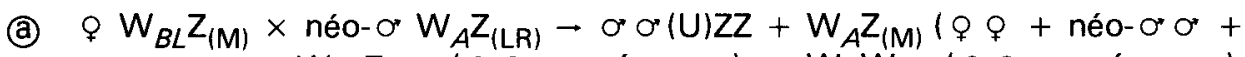

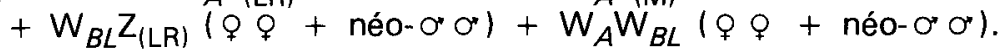

Les rapports $R$ concernant les 4 phénotypes sont relatés dans le tableau 1, ainsi que les valeurs du $\chi^{2}$ (pour 3 d.l.) (test d'homogénéïté pour chaque phénotype coloré). Dans ces croisements, l'expressivité du phénotype A a été normale.

Le taux de néo-mâles observé diffère nettement selon le phénotype de coloration : faible en $A B L(0$ à 21 p. 100) et en $A(0$ à 30 p. 100), il est très élevé $(93 \mathrm{p}, 100)$ en $B L$, où il ne diffère pas significativement de celui observé en $F_{1}$ (1), d'après le test de Mann-Whitney. 
TABLEAU 1

Résultats des croisements : $\mathrm{BL}_{1}$ ba $(\mathrm{M}) \times$ néo- $O \mathrm{~A}$ tr $(\mathrm{LR})$

\begin{tabular}{lccccc}
\hline \multicolumn{1}{c}{ Croisements } & 1 & 2 & 3 & 4 & $\chi^{2}$ \\
\hline $\mathrm{R}_{\mathrm{ABL}}$ & $4 / 15$ & $3 / 57$ & $0 / 14$ & $0 / 44$ & $13,08^{*}$ \\
$\mathrm{R}_{\mathrm{AB}}$ & $0 / 15$ & $18 / 41$ & $0 / 19$ & $5 / 40$ & $15,93^{*}$ \\
$\mathrm{R}^{\mathrm{BL}}$ & $16 / 0$ & $38 / 4$ & $24 / 2$ & $41 / 3$ & 1,62 \\
$\mathrm{R}_{\mathrm{U}}$ & $10 / 0$ & $52 / 0$ & $19 / 0$ & $49 / 0$ & \\
\hline
\end{tabular}

(légende dans le texte). ${ }^{*}: \chi^{2}$ significatifs.

4) Croisements en retour entre $\& \odot$ hybrides $A B L$ et $O^{*} O^{\prime} U$, d'une part, tricuspidata, d'autre part, basteri.

9 femelles $A B L$ apparues en $F_{1}(3)$, dans le croisement $n^{\circ} 2(R=3 / 57)$ ont été croisées, dans un premier temps, avec un mâle $U$ tricuspidata (tr) prélevé dans la population d'Oléron, puis, dans un second temps, avec un mâle $U$ basteri $(b a)$ issu de la lignée $A_{9}$ de Marseille. Seule la femelle $n^{\circ} 5$ (morte dans l'intervalle) n'a donné que des descendances avec le mâle tr. La disjonction en deux phénotypes : $\mathrm{A}$ et $\mathrm{BL}$ chez des individus qui sont tous des femelles génétiques peut être prédite par l'équation :

(b) i $\mathrm{W}_{A} \mathrm{~W}_{B L} \times$ Or $^{\prime} \mathrm{ZZ} \rightarrow \mathrm{W}_{A} \mathrm{Z}+\mathrm{W}_{B L} \mathrm{Z}$.

Il est à noter que, dans toutes ces descendances $\left(\mathrm{CRF}_{2}\right)$, l'expressivité du phénotype $A$ a été réduite uniquement chez les néo-mâles. Les rapports $R$ obtenus sont relatés dans le tableau 2.

4a/ L'analyse statistique des résultats peut d'abord être faite (par ligne) sur l'ensemble des 9 croisements $\left(\chi^{2} \mathrm{~T}\right)$. Elle montre que, en ce qui concerne les $\mathrm{R}_{\mathrm{A}}$ les résultats sont hétérogènes tant avec les mâles $\operatorname{tr}\left(\chi^{2}=24,8-\right.$ d.I. $\left.=8\right)$ qu'avec les mâles ba $\left(\chi^{2}=49,4-\right.$ d.l. $\left.=7\right)$. En ce qui concerne les $R_{B L}$, il n'y a hétérogénéité qu'avec les mâles $\operatorname{tr}\left(\chi^{2}=47,3-\right.$ d.I. $\left.=8\right)$, le $\chi^{2}$ n'étant pas significatif pour les mâles ba $(12,5-$ d.I. $=7)$.

Le test de Mann-Whitney employé pour comparer les deux ensembles montre :

1) que la différence est très significative entre les taux de néo-mâles $A$ et $B L$ obtenus dans les 9 croisements avec les mâles $t r$, le $t_{i} A$ variant de 8,8 à 31,7 p. 100 , les $t_{i} B L$ (beaucoup plus élevés) variant de 48,9 à 84 p. 100 ; la différence reste significative (à la limite) entre les $t_{i} A$ et les $t_{j} B L$ : le $t_{i} A$ varie de 7,4 à 44,6 p. 100 , le $t_{i} B L$ de 28,5 à 54,4 p. 100 dans les 8 croisements avec les mâles $b a$. 2) que les $t_{i} B L$ des croisements utilisant des mâles ba sont significativement plus faibles que ceux obtenus avec les mâles tr. En ce qui concerne les individus $A$, ce même test ne révèle pas de différence globale significative en fonction de l'origine du mâle.

4b/ L'analyse statistique peut être également effectuée à l'intérieur de chaque croisement (par colonne).

1) Avec chacun des mâles tr, la comparaison du $R_{A}$ et du $R_{B L}\left(\chi^{2} R_{A}\right.$ vs $\left.R_{B L}\right)$ (d.I. $=1$ ) montre des différences très significatives, qui confirment le résultat (a1) du test de Mann-Whitney. Avec chacun des mâles $b a$, la différence $\left(\chi^{2} \mathrm{R}_{\mathrm{A}}\right.$ vs $\left.R_{B L}\right)(d . I .=1)$ ne reste significative que dans les croisements $n^{\text {os }} 2,6,7$ et 8 . 


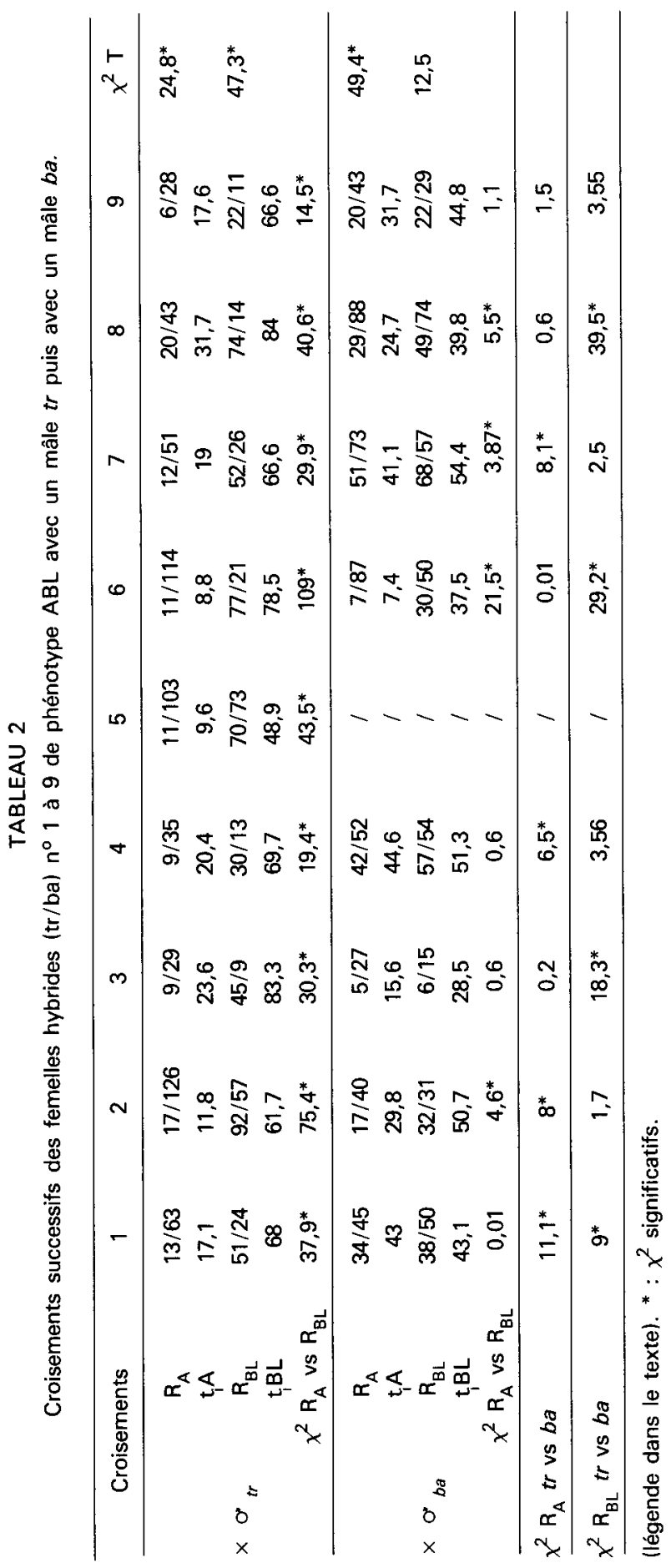


2) Il est également possible de comparer l'évolution des rapports $R$ pour chacune des 8 femelles ayant donné une descendance avec les 2 types de mâles : - en ce qui concerne les $R_{A}\left(\chi^{2} R_{A}\right.$ tr vs ba) (d.I. = 1), la différence est significative (s'accompagnant d'une hausse de $\mathrm{R}$ lorsque l'on passe du mâle tr au mâle ba) dans les croisements $1,2,4$ et 7 ;

- en ce qui concerne les $R_{B L}\left(\chi^{2} R_{B L}\right.$ tr vs $\left.b a\right)(d . I .=1)$, la différence est significative ( $s^{\prime}$ accompagnant d'une baisse de $R$ lorsque la femelle est croisée avec le mâle ba) dans les croisements $1,3,6$ et 8 . Néanmoins les $\chi^{2}$ des croisements 4 et $9(3,56$ et 3,55$)$ sont proches de la significativité.

5) Modalités de l'inversion sexuelle des femelles $W_{A} W_{B L}$.

II est à remarquer que dans les croisements (3) utilisant des néo- $O^{*} O^{*} \mathrm{~A}$ et des $O Q B L$, les taux d'inversion des individus $A B L$ sont très faibles. Ainsi que le montre l'équation (b), ces individus $A B L$ répondent à une constitution WW. Le défaut d'inversion du sexe des $\$$ \& $A B L$ provient-il de leur constitution WW ou de l'association des mutations $A, B$ et $L$ ? Pour étudier ce problème, nous avons effectué 8 croisements entre des néo- $\sigma \sigma^{\circ} B L$ et leurs sœurs, apparus, en (2), dans la descendance $\& \mathrm{BL}_{4} \times \mathrm{O}^{\prime} \mathrm{U}$ rochellais. Ce croisement répond à l'équation :

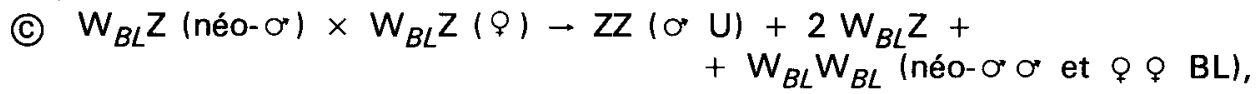

qui fait apparaître $3 / 4$ d'individus $B L$, dont $1 / 3$ sont de formule $W W$.

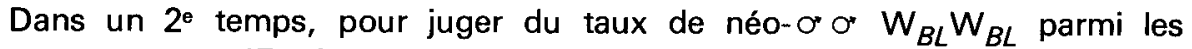
néo- $O^{\prime} O^{*}$ apparus, 17 néo- $O^{*} O^{*} B L$ ont été croisés avec des femelles basteri $U$ marseillaises. Ce croisement répond à 2 équations possibles : les descendances uniquement constituées d'individus $B L$ sont issues des néo- $\sigma^{*} O^{*} W W$, celles qui comportent moitié d'individus $B L$ et moitié d'uniformis proviennent de néo- $O^{\prime}$ or $W_{B L} Z$. Parmi les 17 croisements réalisés, un seul (ayant offert une descendance comportant $15 \mathrm{BL}$ et aucun U) a été du 1er type. Ainsi, sur 17 néo- $\mathrm{O}^{*} \mathrm{O}^{*}$ testés, 1 seul était $W_{B L} W_{B L}$ et la différence est significative $\left(\chi^{2}=5,76\right)$ avec la proportion $1 / 3$ prévue par l'équation (c). Ceci tend à montrer que les femelles $W W$, qu'elles présentent les mutations $A, B$ et $L$, ou simplement les mutations liées $B$ et $L$, s'inversent moins facilement que les femelles WZ.

6) Corrélation entre inversion du sexe et origine du mâle tricuspidata.

Pour tester le rôle du mâle dans l'inversion du sexe des femelles hybrides, ont été effectués des croisements entre les femelles basteri (M) - prises dans les lignées $B L_{1}$ et $B L_{4}$ où toutes les femelles sont favorables à l'inversion lorsqu'elles sont croisées avec des mâles $A_{1}$ rochellais - et des mâles tricuspidata $U$ récoltés à Oléron ou à Pirou. L'exposé des résultats ne fera pas mention des individus $\mathrm{U}$, tous mâles et en nombre conforme à l'équation théorique (proportion $\mathrm{BL} / \mathrm{U}=1$ ).

6a/ Résultats avec les mâles $U$ d'Oléron.

1) Avec les femelles $B L_{1} .4$ femelles de ce type ont été utilisées; elles ont été croisées avec 5 mâles tr $U$ d'Oléron selon le protocole représenté dans le tableau 3, qui donne les $R_{B L}$ obtenus. Il est à remarquer que les croisements successifs de la femelle $n^{\circ} 3$ avec 3 mâles différents aboutissent à des $R$ très hétéro- 
gènes $\left(\chi^{2}=55,9-\right.$ d.I. $\left.=2\right)$; par contre, le croisement de 2 femelles $\left(n^{\circ} 1\right.$ et 2 ) avec le même mâle $\left(n^{\circ} 1\right)$ donne des $R$ homogènes $\left(\chi^{2}=1,7-\right.$ d.I. $\left.=1\right)$. 2) Avec 17 femelles $B L_{4}$, les rapports sont les suivants : $27 / 13$ 21/17 74/30 9/21 9/18 20/26 26/48 16/7 37/32 19/31 36/104 5/13 15/48 15/36 2/15 1/28 0/36. Cet ensemble est très hétérogène $\left(\chi^{2}=143,9-\right.$ d.I. $\left.=16\right)$, le taux d'inversion allant de 0 à 71 p. 100.

\section{TABLEAU 3}

Rapports $\mathrm{R}_{\mathrm{BL}}$ obtenus dans les croisements entre mâles $\operatorname{tr} U$ d'Oléron et femelles ba $(\mathrm{M}) \mathrm{BL}$,

\begin{tabular}{|c|c|c|c|c|c|}
\hline \multicolumn{6}{|c|}{$\sigma^{\circ}$} \\
\hline & 1 & 2 & 3 & 4 & 5 \\
\hline 1 & $46 / 14$ & 1 & I & 1 & 1 \\
\hline 2 & $7 / 6$ & I & I & 1 & 1 \\
\hline 3 & 1 & $53 / 0$ & $51 / 25$ & $25 / 46$ & 1 \\
\hline 4 & 1 & 1 & 1 & 1 & $0 / 56$ \\
\hline
\end{tabular}

6b/ Résultats avec les mâles de Pirou.

Ils ont été testés avec 16 femelles de la lignée $\mathrm{BL}_{4}$ (différentes de celles utilisées en 6.a2) ; les rapports $R_{B L}$ ont été : $33 / 26 \quad 7 / 13 \quad 8 / 24 \quad 11 / 21 \quad 27 / 32$ 15/42 $16 / 3020 / 4 \quad 47 / 4 \quad 14 / 0 \quad 29 / 52 / 7424 / 522 / 10 \quad 33 / 33$ 48/18. L'ensemble est très hétérogène $\left(\chi^{2}=207,6-\mathrm{d} . \mathrm{l}\right.$. $\left.=15\right)$, avec un taux d'inversion allant de 2,6 à 100 p. 100.

Le test de Mann-Whitney révèle que le taux des néo-mâles résultant des croisements entre mâles de Pirou et femelles $\mathrm{BL}_{4} \mathrm{n}^{\prime}$ est pas significativement plus élevé que celui des croisements entre ces mêmes femelles et les mâles d'Oléron, mais très proche de la significativité.

7) $F_{2}$ issues des deux types de $F_{1}$ défavorables à l'inversion du sexe.

Nous avons rencontré, au cours des croisements précédents entre les sousespèces basteri et tricuspidata, deux types de $F_{1}$ dépourvues de néo-mâles ou n'en présentant qu'un taux très faible:

- des croisements employant des mâles tricuspidata pris en lignée $A_{1}$ de La Rochelle que l'on peut considérer comme favorables à l'inversion sexuelle de leurs filles, mais offrant néanmoins dans leur descendance une absence totale, ou presque, de néo-mâles lorsqu'ils sont croisés avec certaines femelles basteri BL de Marseille ou du Racou ;

- des croisements entre des femelles basteri prises dans des lignées favorables à l'inversion ( $B L_{1}$ et $B L_{4}$ de Marseille) lorsqu'elles sont croisées avec des mâles rochellais, mais qui engendrent des descendances dépourvues de néo-mâles lorsqu'elles sont croisées avec certains mâles tricuspidata d'Oléron ou de Pirou. 
La variabilité du taux d'inversion en $F_{2}$ peut contribuer à nous renseigner sur les rôles respectifs des génotypes mâles et femelles dans le déterminisme de ce phénomène.

7a/ $F_{2}$ des croisements : $\subsetneq$ basteri $\mathrm{BL}$ du Racou $\times$ o rochellais.

1) $F_{2}$ du croisement : $\subsetneq \mathrm{BL}_{1}$ du Racou $\times \circlearrowleft \mathrm{U}$ La Rochelle. La $F_{1}$ avait donné un rapport $\mathrm{R}=0$ néo- $\sigma / 30 \subsetneq \wp \mathrm{BL}$. Sur $16 \mathrm{~F}_{2}$ retenues (nombre de descendants $>10), 11$ n'ont donné aucun néo-mâle $(0 / 250 / 30 \quad 0 / 110 / 140 / 160 / 32$ $0 / 110 / 160 / 430 / 210 / 43)$. Les cinq autres en ont donné chacun un $(1 / 611 / 39$ $1 / 321 / 331 / 31$ ).

2) $\mathrm{F}_{2}$ du croisement : $\odot \mathrm{BL}_{3}$ du Racou $\times \sigma^{*} \mathrm{U}$ La Rochelle. La $\mathrm{F}_{1}$ avait donné : 0 néo- $O^{*} / 18$ \& $\mathrm{BL}$. Sur $9 F_{2}$ retenues, une seule $n^{\prime}$ offre aucun néo- $\sigma^{*}(0 / 33)$, les 8 autres en présentent: $R=1 / 281 / 291 / 391 / 37$ 1/36 1/26 1/12 4/42. 7b/ $\mathrm{F}_{2}$ du croisement: \& basteri $\mathrm{BL}_{4}(\mathrm{M}) \times \mathrm{o}^{\prime}$ tricuspidata (Pirou).

\section{TABLEAU 4}

Répartition des classes de $t_{i}$ dans les 3 séries de $F_{2}$ réalisées au paragraphe 7

\begin{tabular}{cccc}
\hline Classes de $t_{i}$ & \multicolumn{3}{c}{ Croisements } \\
\cline { 2 - 4 } & $7 a_{1}$ & $7 a_{2}$ & $7 b$ \\
\hline 0 p. 100 & 11 & 1 & 7 \\
\hline 1 à 5 p. 100 & 5 & 6 & 3 \\
\hline$>5$ p. 100 & 0 & 1 & 12 \\
\hline
\end{tabular}

A partir d'une $F_{1}$ ayant abouti à un rapport : 2 néo- $\sigma^{\prime} \sigma^{\prime} / 74 \%$ $Q \mathrm{BL}\left(\mathrm{t}_{\mathrm{i}}=2,6\right.$ p. 100), sur $22 F_{2}$ retenues, 7 n'offrent aucun néo-mâle $10 / 340 / 360 / 690 / 15$ $0 / 220 / 140 / 15)$; les autres en comportent de façon variable: $1 / 58$ 1/38 2/40 $1 / 204 / 665 / 656 / 722 / 233 / 224 / 286 / 405 / 245 / 135 / 125 / 6$. Ces résultats forment un ensemble fortement hétérogène $\left(\chi^{2}=79,6-\right.$ d.I. $\left.=21\right)$, le taux d'inversion allant de 0 à 45 p. 100.

La comparaison des résultats de ces 3 séries de $F_{2}$ a été effectuée en classant les $t_{i}$ observés en 3 groupes : $t_{i}=0, t_{i}$ de 1 à $5 \%, t_{i}>5 \%$. Ce classement donne la répartition figurée dans le tableau 4.

Cet ensemble est hétérogène $\left(\chi^{2}=22,87-d . I\right.$. $\left.=4\right)$. Les $F_{2} 7 a 1$ et $7 a 2$ diffèrent l'une de l'autre $\left(\chi^{2}=7,60-\right.$ d.I. $\left.=2\right)$; il en est de même des $F_{2} 7 \mathrm{a} 2$ et $7 \mathrm{~b}\left(\chi^{2}=10,58\right)$ et des $F_{2} 7 \mathrm{a} 1$ et $7 \mathrm{~b}\left(\chi^{2}=12,76\right)$.

\section{Discussion et conclusion.}

- Du fait de la constance du taux (27 p. 100) d'inversés que présente sa descendance en inbreeding, la lignée $A_{1} d^{\prime} l$. balthica tricuspidata de La Rochelle peut être considérée comme ayant un génotype favorable à l'inversion sexuelle d'une fraction importante des femelles. 
- Le taux très élevé d'inversions du sexe offert par les femelles hybrides issues du croisement: $q$ q basteri issues des lignées $B L_{1}$ et $B L_{4}(M) \times \sigma$ rochellais peut être également rapporté à un caractère transmissible, présent dans ces deux lignées marseillaises, mais paraissant indépendant des mutations bilineata-lineata (BL) puisque, en (2), de nombreuses femelles basteri BL $(18$ sur 20) de la même population - ou d'une population voisine, croisées avec des mâles rochellais, ne donnent naissance à aucun néo-mâle.

- Réciproquement, les résultats des croisements (6) montrent en fait que l'inversion sexuelle des femelles hybrides résultant du croisement : $₹$ basteri (M) $\left(B L_{1}\right.$ ou $\left.\mathrm{BL}_{4}\right) \times \mathrm{O}^{\prime}$ tricuspidata ne dépend pas d'un génotype exceptionnel offert par la lignée $A_{1}$ rochellaise, mais d'une constitution quasi générale chez les individus de la sous-espèce tricuspidata : effectivement, si l'on ajoute aux 22 mâles d'Oléron testés en (6) les 9 mâles de même origine utilisés en (4), ce sont 28 mâles sur 31 (90 p. 100) qui se sont montrés favorables à l'inversion sexuelle des femelles hybrides, et 15 sur 16 (94 p. 100) à Pirou.

- Les croisements (5) mettent en évidence le rôle du chromosome $W$ dans l'inversion du sexe et, en particulier, du (ou des) gène(s) porté(s) par le segment impair. En effet, rien ne distinguerait les femelles WW et WZ en matière d'inversion du sexe si entraient uniquement en jeu des gènes portés par le segment pair. Par ailleurs, la faiblesse $d u t_{i}$ des individus $A B L$, observée en (3), est bien due à une constitution WW : effectivement, lorsque sont effectués, en (4), des croisements aboutissant à la disjonction des deux chromosomes $W$, le $t_{i}$ redevient notable, aussi bien chez les individus $A$ que chez les $B L$. Ces données appuient la conception que $W$ porte, sur son segment impair, un déterminant sexuel femelle majeur, dont une double dose ne s'oppose pas d'une façon absolue à l'inversion sexuelle de la " super femelle ", mais en restreint fortement les possibilités.

- Les deux séries de $\mathrm{CRF}_{2}$ effectuées en (4) montrent également que la structure du chromosome $W$ et (ou) la pénétrance des gènes qu'il porte module(nt) le taux des néo-mâles :

a) On observe chez les individus $A$ et $B L$ nés d'un même père une corrélation entre taux de néo-mâles (qui est généralement plus élevé chez les $B L$ ) et origine subspécifique du $W$ marqué par la mutation strictement liée au sexe. En effet, la constitution chromosomique de ces deux types de femelles scurs ne diffère, en principe, que par l'origine du $W$ et la mutation qu'il porte : tricuspidata chez les mutants albafusca, basteri lorsque $W$ est marqué par les mutations $B L$ (le reste du génotype étant en grande partie fonction du mâle utilisé : tricuspidata ou basteri, qui fournit le chromosome $Z$, un lot haploïde complet d'autosomes, le lot complémentaire d'autosomes provenant de la femelle hybride : $\operatorname{tr} / b a)$.

On retrouve le même processus dans des croisements - qui n'ont pas été développés ici - entre des néo-mâles hybrides $W_{A(L R)} Z_{(M)}$ (où la pénétrance de $A$ est complète) et des femelles basteri $\mathrm{BL}_{1(\mathrm{M})}$; par exemple, un de ces croisements a donné les rapports $R: 2 / 69$ en $A B L, 0 / 74$ en $A, 22 / 34$ en $B L$, $62 / 4$ en $U$ (les 4 femelles $U$ apparues étant des néo-femelles $Z Z$ ). Les femelles $B L$ et $A$ ne diffèrent aussi, en principe, que par l'origine du $W$ et la mutation 
qu'il porte : tricuspidata pour les $A$, basteri pour les $B L$, or les taux d'inversion des deux phénotypes sont très différents. Il est d'ailleurs impossible, dans ces résultats, de distinguer la part qui revient, d'une part, à l'origine subspécifique du W, d'autre part, aux mutations : $B L$ ou $A$, dans la différence des taux d'inversion.

b) Les résultats de tous les $C_{2} F_{2}$ (4) montrent que le défaut d'expressivité du phénotype $A$, c'est-à-dire la pénétrance incomplète du gène $A$, se produit uniquement chez les néo-mâles; autrement dit, il s'accompagne toujours de l'inversion sexuelle de la femelle hybride qui le présente. De ce fait, le $t_{i A} n$ 'est jamais nul [alors qu'il peut l'être, en (3), lorsque la pénétrance de $A$ est complète], et dépasse assez fréquemment 30 p. 100 . Ceci confirme donc les résultats que nous avons obtenus dans le travail précédent (1982, sous presse).

La corrélation étroite entre pénétrance du gène $A$ et inversion sexuelle suggère que ce gène est impliqué dans la détermination du sexe : il pourrait être situé sur le segment d'ADN correspondant au déterminant femelle majeur porté par le segment impair de $W$ : lorsque ce locus est occupé par le gène $A^{+}$(ce qui intervient, entre autre, chez les femelles $B L$ ), il serait plus favorable à l'inversion que dans l'état $A$; lorsque la pénétrance de $A$ est incomplète, l'effet du gène tendrait à être comparable à celle de $A^{+}$.

c) L'affaiblissement de la pénétrance de $A$ n'ayant été observé que dans certains croisements et chez des hybrides présentant un $W_{A}$ d'origine tricuspidata il peut dépendre soit du $W$ de cette sous-espèce, soit du complexe génique hybride porté par $Z$ et (ou) les autosomes, soit de l'une et de l'autre de ces composantes du génotype hybride.

$c_{1}$ ) La structure du $W_{A}$ doit être impliquée dans ce processus. En effet, si l'on part d'une $\mathrm{F}_{1}$ : ᄋ A basteri $\times$ O’ $\mathrm{U}$ tricuspidata, croisement où la pénétrance de $A$ est toujours complète, le renforcement du génotype tricuspidata ou basteri par des croisements en retour des femelles hybrides avec des mâles de l'une ou I'autre sous-espèce ne modifie en rien l'expressivité du phénotype $A$, ainsi que nous l'avons montré dans le précédent travail (1982). Ceci suppose l'existence d'un obstacle majeur à une baisse de la pénétrance de $A$, qui ne peut être rapporté qu'au chromosome $W_{A}$. Cette conclusion rejoint celle du point (a) de cette discussion, en précisant toutefois qu'il s'agit bien d'une différence subspécifique dans la structure de $W$ et non pas causée par la mutation qu'il porte.

$\mathrm{C}_{2}$ ) En ce qui concerne l'action des gènes portés par $Z$ et (ou) les autosomes, la comparaison des $t_{i}$ des individus $A$ et $B L$ lorsque l'on croise en (4), successivement une même femelle avec deux mâles, I'un tricuspidata, l'autre basteri, montre que les variations significatives de ces taux (variations qui sont d'ailleurs de sens inverse) ne se produisent généralement pas dans une même descendance. Les hétérochromosomes $W$ conservant strictement la même structure grandmaternelle dans les deux séries de croisements (puisque $W_{B L}$ porte un suppresseur de crossing-over), c'est donc du changement du reste du génotype que dépendent les variations des $t_{i}$. Ce ne sont donc généralement pas les mêmes complexes géniques portés par $Z$ et (ou) les autosomes qui sont favorables, respectivement, à l'inversion du sexe accompagnée d'une baisse d'expressivité de $A$, et à l'inversion du sexe des femelles hybrides présentant un $W$ porteur de $B L$. 
Cette étude des croisements entre /dotea balthica tricuspidata et $/$. b. basteri, en utilisant comme marqueurs de l'hétérochromosome $\mathrm{W}$ les mutations $B L$ et $A$, apporte ainsi des résultats comparables à ceux obtenus dans notre travail précédent (1982), conduisant à la conclusion que le déterminisme de l'inversion du sexe femelle fait intervenir un ensemble complexe de corrélations géniques entre, d'une part, le segment impair de l'hétérochromosome $W$ et, d'autre part, des gènes pairs partiellement liés au sexe et (ou) autosomiques; l'intervention d'un segment impair du chromosome Z, qui n'a pu être jusqu'ici démontrée génétiquement ou cytologiquement, ne peut être cependant écartée.

Le fait que l'origine subspécifique du chromosome $W$ et la pénétrance du gène $A$, selon que cette dernière est complète ou incomplète, retentissent sur l'inversion du sexe peut se rattacher à la notion de valence sexuelle introduite par Goldschmidt (1955) chez Lymantria dispar et reprise par d'autres auteurs, notamment Kallman (1965) chez Xiphophorus maculatus, Legrand et al. (1980) chez Porcellio dilatatus. Quant à l'intervention d'un complexe autosomique dans la détermination du sexe, elle a été invoquée par maints auteurs, en particulier: De Lattin (1958), chez différents Oniscoïdes, Winge et Ditlevson (1948), Hammerman et Avtalion (1979) chez les poissons. L'action de gènes autosomiques est bien connue également chez Drosophila (références in Baker et Ridge, 1979).

La réalisation, chez les Oniscoïdes, chez Orchestia gammarellus et Idotea balthica, d'une inversion expérimentale du sexe par implantation d'une glande androgène chez une jeune femelle (références in Legrand et Juchault, 1970 ; Ginsburger-Vogel, 1972 ; Legrand-Hamelin, 1977) permet de mieux poser le problème de la détermination du sexe. Il a été en effet montré, chez les Oniscoïdes et Orchestia, que le néo-mâle obtenu différencie une néo-glande androgène fonctionnelle. La femelle possède donc le territoire et les gènes nécessaires à la sécrétion de l'hormone mâle. Ainsi, ce qui lui manquait - et que lui apporte, dans un premier temps, l'hormone mâle allochtone - pourrait être une dérépression des gènes mâles. Le fait que l'inversion spontanée des femelles WW d'/dotea est moins fréquente que celle des femelles WZ est à rapprocher des résultats obtenus chez Porcellio dilatatus (Legrand et al., 1980), où l'inversion expérimentale des femelles $X Z$ est beaucoup plus rapide que celle des femelles $X X$ (4 à 5 mois contre 12 mois). Ces diverses données sont ainsi à l'appui de l'hypothèse que $W$ (ou $X$ ) contient un répresseur des gènes mâles, dont une double dose assure une répression plus forte. Une partie importante des gènes sexuels mâles peut être située sur $Z$ : cette hypothèse contribuerait à expliquer la rapidité de l'inversion expérimentale des femelles $X Z$ chez Porcellio, la plus grande fréquence d'inversion spontanée des femelles WZ d'/dotea. Cependant, du fait que les femelles WW ou $X X$ peuvent également s'inverser, il faut bien admettre que les autosomes - et peut être le segment pair de $W($ ou $X)$ - contiennent également des gènes mâles. Réciproquement, l'existence de néo-femelles $Z Z$ conduit à admettre la présence de gènes sexuels femelles autosomiques - et peut être partiellement liés à $Z$ - , ainsi que l'a déjà fait remarquer l'un de nous (TinturierHamelin, 1963). Ceci rejoint le fait que chez Armadillidium vulgare, les mâles homogamétiques $(Z Z)$ peuvent être féminisés par le bactéroïde $F$ (références in Legrand, 1977) : ils possèdent donc les gènes nécessaires à la différenciation et 
au fonctionnement d'un ovaire (sécrétion d'hormone femelle) ; ces gènes se mettent à fonctionner si la différenciation de la glande androgène est inhibée : c'est le processus de l'autodifférenciation ovarienne (Charniaux-Cotton, 1962 ; Juchault et Legrand, 1964).

Il est possible, à la suite de la remarque précédente, de concevoir que la différenciation sexuelle dépende de la présence (ou de l'absence) du déterminant mâle contrôlant la différenciation de la glande androgène (Charniaux-Cotton, 1965). Mais ce schéma simple, rapporté par cet auteur à un seul couple d'allèles (à l'instar de ce qui a été proposé par Gilchrist et Haldane, 1947, chez Culex molestus) ne rend compte ni de la complexité des phénomènes d'inversion du sexe ni de la difficulté de s'inverser offerte par les femelles WW ou XX, à I'inverse des femelles WZ d'/dotea ou XZ de Porcellio.

Le schéma auquel l'ensemble des résultats exposés ici aboutit est celui d'un déterminisme polyfactoriel du sexe, dont le fondement paraît être, dans chaque sexe, une répression des gènes, encore inconnus, conditionnant l'ensemble des caractères sexuels de l'autre sexe. Cette répression peut se réaliser de différentes façons. Chez un mâle génétique d'Oniscoïde, c'est l'inducteur sexuel mâle de la gonade larvaire (Juchault et Legrand, 1964), puis I'hormone androgène circulante qui répriment les gènes contrôlant la différenciation sexuelle, respectivement primaire et secondaire femelle. Bien qu'ayant évidemment une base génétique, cette répression ne découle que secondairement de l'action des gènes contrôlant la différenciation de la glande androgène. Chez une femelle génétique, il $n$ 'en est pas ainsi : I'hormone ovarienne n'est synthétisée qu'à partir de la puberté. II faut donc que la répression des gènes de la différenciation sexuelle mâle soit d'origine génétique directe, c'est-à-dire probablement un produit primaire du répresseur situé sur le segment différentiel de $W$. Ce répresseur peut être considéré comme un des déterminants majeurs du sexe femelle.

Bien que les néo-femelles ZZ n'aient pas fait, ici, l'objet d'une étude systématique, pour expliquer leur réalisation on peut admettre l'existence de gènes modificateurs autosomiques et (ou) partiellement liés au sexe, capables d'inhiber les déterminants mâles, et ainsi de pallier l'absence du répresseur porté par $W$.

Les résultats relatés ici et dans notre publication précédente (1982) montrent que, chez Idotea balthica, le déterminant femelle de $W$ est modulé à son tour par deux complexes de modificateurs portés par $Z$ et (ou) les autosomes. La figure 1 récapitule ces hypothèses dans le cas de la réalisation d'un néo-mâle WZ.

Chez la sous-espèce I. b. basteri, l'action des modificateurs de $W$ se fait très exceptionnellement sentir, ce qui peut être rapporté à un état allélique dominant défavorable, ainsi qu'à une valence généralement forte du répresseur porté par $W$.

Cette conclusion s'accorde avec la rareté des néo-mâles dans les populations naturelles de cette sous-espèce, et dans la grande majorité des $F_{1}(18 / 20)$ : ○ basteri (BL) $\times$ ơ tricuspidata $U$ effectuées en (2).

Les données obtenues en $\mathrm{F}_{2} 7 \mathrm{a}_{1}$ et $7 \mathrm{a}_{2}$ montrent que, partant de deux de ces $F_{1}$ dépourvues de néo-mâles, le $t_{i}$ nul ou très faible persiste, ce qui semble indiquer l'existence d'une inhibition quasi absolue de l'inversion sexuelle que l'on peut rapporter à une valence élevée du $W$ de la femelle basteri, dont héritent 




FIG. 1. - Schéma résumant les hypothèses concernant la réalisation d'un néo-mâle WZ.

S.i. et S.p. : segments impair et pair des hétérochromosomes; G.A. : glande androgène ; H.A. : hormone androgène ; $(1)$ : modificateurs agissant sur la pénétrance de $A ;(2)$ : modificateurs n'agissant pas sur la pénétrance de $A$. En traits continus : systèmes actifs; en traits discontinus : systèmes inactifs.

toutes les filles et petites-filles. Les rares néo-mâles seraient dus à la conjonction de modificateurs favorables à l'inversion, parvenant à atténuer la valence forte de $W$, ce qui doit être plus fréquent chez une femelle hybride descendant d'un géniteur de la $F_{1}$ favorable, que dans une population naturelle, où, effectivement, les $t_{i}$ sont un peu plus faibles que ceux observés dans ces $F_{2}$.

Au contraire, partant d'une $F_{1}$ à taux presque nul de néo-mâles $(R=2 / 74)$, obtenue par croisement d'un mâle tricuspidata de Pirou avec une femelle basteri, prise dans une lignée favorable à l'inversion $\left(B L_{4} M\right)$, le taux de néo-mâles dans les $F_{2} 7 b$ s'échelonne entre 0 et 45 p. 100 . Ceci peut s'expliquer en admettant : a) que les femelles $B L_{4}(M)$ offrent, d'une part, une valence faible du répresseur porté par W, d'autre part, une conjonction des gènes modificateurs défavorable à l'inversion, qui rend compte de l'absence de néo-mâles en inbreeding et du $t_{i}$ très faible obtenu dans la $F_{1}$ avec un ơ également défavorable ;

b) que ce serait la réalisation, en $F_{2}$, de recombinaisons des modificateurs tricuspidata-basteri qui permettrait l'apparition de taux notables de néo-mâles.

Chez l. b. tricuspidata, l'existence fréquente de néo-mâles dans les populations naturelles suggère une valence faible du répresseur que porte $W$ et un état hétérozygote des gènes modificateurs.

Quelle peut être l'incidence, pour la survie de la sous-espèce tricuspidata, de cette possibilité d'inversion du sexe : accroît-elle le taux de mâles ? En fait, il $\mathrm{n}^{\prime}$ en est probablement rien : la sex-ratio, dans la nature, est normale (exemple : $410^{\circ} 0^{\prime \prime}, 44 \bigcirc$ ㅇ récoltés à Pirou en août 1980). Les mâles sont, d'une part, plus fragiles que les femelles (en élevage), d'autre part, le croisement d'un néo-mâle et d'une femelle génétique donne une sex-ratio théorique de $10^{\circ} / 3 \%$ avec, parmi celles-ci, des femelles thélygènes (WW). Il est donc possible, en définitive, que la formation de néo-mâles aboutisse à un rééquilibrage de la sex-ratio au 
cours des générations. C'est plutôt la formation de néo-femelles (ZZ) qui conduirait à une arrhénogénie dès la $F_{2}$, mais le taux de ces néo-femelles est très faible dans les populations naturelles étudiées ici. Ce problème de génétique dès populations sera analysé ultérieurement.

La Méditerranée actuelle s'étant repeuplée à partir de l'Atlantique après une période d'isolement et de sursalinité, il est probable qu'l. b. tricuspidata est proche de l'espèce ancestrale et qu' $\%$. b. basteri en dérive. La détermination génétique du sexe, telle qu'elle se présente dans les deux sous-espèces, semble refléter cette filiation : elle est plus primitive, car non absolue chez tricuspidata, beaucoup plus rigoureuse chez basteri.

On peut concevoir, en reprenant les conclusions de la présente étude, que l'évolution ayant conduit à basteri résulte de la sélection d'une valence forte du déterminant femelle de $W$ et (ou) de gènes modificateurs dominants défavorables à l'inversion des femelles.

Reçu en février 1982

Accepté en mai 1982

\section{Références}

BAKER B. S., RIDGE K. A., 1979. Sex and the single cell. I. On the action of major loci affecting sex determination in Drosophila melanogaster. Genetics, 94, 383-423.

CHARNIAUX-COTTON H., 1962. Déterminisme de l'intersexualité chez les Crustacés supérieurs, et particulièrement chez les Amphipodes Ta/itridae. Bull. Soc. Zool. Fr., 87, 338-336.

CHARNIAUX-COTTON H., 1965. Contrôle endocrinien de la différenciation sexuelle chez les Crustacés supérieurs. Arch. Anat. microsc. Morphol. expér., 54, 1, 405-415.

FAVERGE J. M., 1962. Méthodes statistiques en psychologie appliquée. P.U.F., Paris.

GILCHRIST B. M., HALDANE J. B. S., 1947. Sex linkage and sex determination in a mosquito, Culex molestus. Hereditas, Lund, 33, 175-190.

GINSBURGER-VOGEL T., 1972. Inversion des femelles d'Orchestia gammarella Pallas (Crustacés Amphipodes Talitridae) en néo-mâles fonctionnels par greffe de glandes androgènes avant la mue de première différenciation externe du sexe. C.R. Acad. Sci. Paris, sér. D, 274, 3606-3609.

GOLDSCHMIDT R. B., 1955. Theoretical Genetics. Univ. California Press, Berkeley and Los Angeles.

HAMMERMAN I. S., AVTALION R. R., 1979. Sex determination in Sarotherodon (Tilapia). II : The sex-ratio as a tool for the determination of genotype. A model of autosomal and gonosomal influence. Theor. appl. Genet., 55, 177-187.

JUCHAULT P., LEGRAND J. J., 1964. Mise en évidence d'un inducteur sexuel mâle distinct de l'hormone adulte et contribution à l'étude de l'auto-différenciation ovarienne chez l'Oniscoïde Helleria brevicornis. C.R. Acad. Sci. Paris, 258, 2416-2419.

KALLMAN K. D., 1965. Genetics and geography of sex determination in the poeciliid fish, Xiphophorus maculatus. Zoologica, 50, 151-190.

LATTIN G. De, 1958. Vererbungsstudien an Isopoden. I. Zur Genetik der Farbvariafilität von Cylisticus convexus (De G.). Zool. Anz., 160, 313-324.

LEGRAND J. J., 1977. Détermination génétique et épigénétique du sexe chez les Crustacés Isopodes terrestres. Corrélation entre monogénie et intersexualité. Bull. Soc. Sci. nat. Tunisie, 12, 3-12. 
LEGRAND J. J., JUCHAULT P., 1970. Le contrôle humoral de la sexualité chez les Crustacés Isopodes gonochoriques. Cours et Documents de Biologie, 3, 178-218. Gordon et Breach Paris-Londres-New York.

LEGRAND J. J., JUCHAULT P., MOCQUARD J. P., MARTIN G., 1980. Polymorphisme d'origine géographique portant sur la « valence " des chromosomes sexuels et phénomènes de monogénie résultant du croisement de différentes populations de Porcellio dilatatus Brandt (Crustacé Isopode terrestre). Reprod. Nutr. Dévelop., 20, 23-59.

LEGRAND-HAMELIN E., 1977. Obtention de néo-mâles fonctionnels et démonstration expérimentale de l'hétérogamétie femelle chez ldotea balthica (Crustacé Isopode). C.R. Soc. Biol., 171, 176-180.

LEGRAND-HAMELIN E., LEGRAND J. J., 1982. Déterminisme génétique de l'inversion du sexe femelle accompagnant le croisement de deux sous-espèces d'ldotea balthica (Pallas). I. Résultats concernant les phénotypes albafusca, bilineata et uniformis. Reprod. Nutr. Dévelop., 22, 753-766.

TINTURIER-HAMELIN E., 1963. Polychromatisme et détermination génétique du sexe chez l'espèce polytypique /dotea balthica (Pallas) (Isopode Valvifère). Cah. Biol. mar., 4, 473-591.

WINGE Ö., DITLEVSON E., 1948. Colour inheritance and sex determination in Lebistes. C.R. Lab. Carlsberg, Ser. Physiol., 24, 227-248. 